\title{
Swimming Upstream: Disentangling the Association between Alcohol Intake and Venous Thromboembolism
}

\author{
Søren Paaske Johnsen ${ }^{1}$ Lars Frost ${ }^{20}$ \\ ${ }^{1}$ Danish Center for Clinical Health Services Research, Department of \\ Clinical Medicine, Aalborg University, Aalborg, Denmark \\ 2 Diagnostic Centre, University Research Clinic for Innovative Patient \\ Pathways, Silkeborg Regional Hospital, Silkeborg, Denmark
}

Thromb Haemost 2019;119:858-859.

Venous thromboembolism (VTE) is a major cause of cardiovascular death and morbidity, and many management advances are evident. ${ }^{1}$ As the incidence of VTE has increased in the past decades, the public health and economic impact of the disease continues to grow despite advances in prophylaxis and treatment. ${ }^{2}$ There is consequently considerable scientific and policy interest for improvement in prevention strategies as well as identification of high-risk patients. ${ }^{3}$ However, seeking upstream in the causal pathway for targets of effective prevention initiatives requires an advanced understanding of the role of important risk factors.

Alcohol intake is for many reasons an interesting candidate as a potential target for VTE prevention strategies. Alcohol is ubiquitous globally and according to the World Health Organization responsible for three million deaths every year worldwide corresponding to $5.3 \%$ of all deaths. ${ }^{4}$ Harmful use of alcohol is considered to be a causal factor in more than 200 diseases and injury conditions. ${ }^{4}$ In addition, four decades of epidemiological studies have studied the possible link between alcohol intake and risk of cardiovascular disease, primarily ischemic heart disease, and have largely found the same J-shaped pattern with a small increase in cardiovascular risk among abstainers, a lower risk for light to moderate drinkers, and a markedly increased risk for abusers. ${ }^{5}$ However, the picture appears a bit more blurred when we try to focus on the association between alcohol intake and VTE. Despite numerous studies, consensus on the role of alcohol remains to be established due to the inconsistent and in some instances apparently contradictory findings. ${ }^{5}$

In this issue of Thrombosis and Haemostasis, Johansson et $\mathrm{al}^{6}$ adds another piece to the puzzle with their large cohort study from Northern Sweden which investigates the longterm association between alcohol consumption, alcohol dependence, and VTE. In short, the authors found an association between increasing alcohol intake and higher risk of VTE

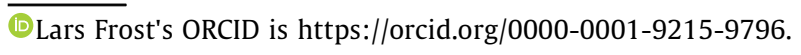

received

March 22, 2019

accepted

March 22, 2019

Address for correspondence Søren Paaske Johnsen, MD, PhD, Danish Center for Clinical Health Services Research, Department of Clinical Medicine, Aalborg University, Mølleparkvej 10, 9000 Aalborg, Denmark (e-mail: spj@dcm.aau.dk).

among men but not among women. Alcohol dependence was associated with the highest risk for VTE in men (adjusted hazard ratio: 1.30; 95\% confidence interval: 1.07-1.59), whereas no clear association was found among women.

The study by Johansson et al comes with several methodological strengths: It is based on prospectively collected data on a large population (more than 100,000 individuals), which appears to reflect the underlying source population well (participation rate $\sim 70 \%$ ). In addition, the study includes detailed data on alcohol intake, virtually complete follow-up, and the more than 2,000 identified first-time VTE events were all validated via review of radiology reports and/or medical records. The limitations were few, but should be noted, including the long follow-up time (median: 13.9 years) without repeated assessment of alcohol intake, which increase the risk of misclassification of the exposure as individuals may change intake over time, lack of data on drinking pattern, including binge drinking, and the lack clinical details regarding the VTE events, including VTE provoking factors.

A higher risk of VTE among individuals with a high alcohol intake has also previously been reported, although not specifically for men. ${ }^{7}$ However, other recent studies have reported null findings or found indications of J- or U-like associations with no clearly increased risk among individuals with a relatively high alcohol intake. ${ }^{8-11}$ The reasons for the apparent discrepancies are multiple and include differences in the assessment of alcohol intake (including possible cultural specific differences in the validity of the selfreported information), reference groups (abstainers vs. individuals with low intake, typically up to one unit per week), covariates included as potential confounding factors in the multivariable analyses, and differences in the detection and assessment of VTE events during follow-up. Hence, it is no surprise that direct comparisons between studies are difficult, as the studies do not address the same questions. The challenges that face researchers studying the association between alcohol intake and VTE are closely linked with the

(c) 2019 Georg Thieme Verlag KG Stuttgart · New York
DOI https://doi.org/ $10.1055 / \mathrm{s}-0039-1687881$. ISSN 0340-6245. 
complexity of the association. Hence, we do not fully understand the mediating factors that may explain an association. Is the potential association mainly explained by direct physiological and biochemical effects of alcohol on the triad of Virchow (i.e., hypercoagulability, hemodynamic changes, and endothelial injury) or the behavior that may follow (or at least be associated with) alcohol intake, including fall, injuries, and an overall more risky lifestyle for individuals with a high intake? The mediating factors may potentially also affect the likelihood of detecting VTE, in particular for patients dying from VTE without a previous hospital diagnosis or for patients with subclinical VTE events.

So, where does this leave us now with respect to alcohol consumption and VTE? We are in an era with increasing hype about "precision public health," which is predicted to ensure gains in public health in the near future by using a "big data" approach and combining detailed individual-level lifestyle, genomic, and physiological data to guide targeted prevention strategies. ${ }^{12}$ However, it is not possible with the current lack of understanding on the impact of alcohol intake on VTE risk to develop such advanced preventive strategies. Still, this should not lead to a nihilistic view on the possibilities for VTE prevention. It is established without any doubt that alcohol is a potentially addictive drug with serious adverse effects on multiple other organ systems, including the cardiovascular system. ${ }^{13}$ Moreover, the harmful use of alcohol brings significant social and economic losses to individuals and society at large. ${ }^{4}$ The outstanding uncertainty in particular on the potential benefits of light-to-moderate drinking for prevention of VTE is in this context of minor importance. Even if light-to-moderate alcohol intake can lower the risk of VTE by 10 to $30 \%$ as suggested by some studies, there is no evidence to suggest that alcohol abstinence is an important public health threat, in particular in Western countries where the prevalence of alcohol abstainers is very low. ${ }^{9,10}$ Rather there is an abundance of evidence to support that our efforts should focus on preventing alcohol abuse and the current study by Johansson et al just adds further evidence to this strategy.
Conflict of Interest

None declared.

\section{References}

1 Schulman S, Ageno W, Konstantinides SV. Venous thromboembolism: past, present and future. Thromb Haemost 2017;117(07): 1219-1229

2 Münster AM, Rasmussen TB, Falstie-Jensen AM, et al. A changing landscape: temporal trends in incidence and characteristics of patients hospitalized with venous thromboembolism 2006-2015. Thromb Res 2019;176:46-53

3 Albertsen IE, Nielsen PB. Searching for high-risk venous thromboembolism patients using risk scores: adding to the heap or closing a gap? Thromb Haemost 2018;118(10):1686-1687

4 Global Status Report on Alcohol and Health 2018. Geneva: World Health Organization; 2018. Licence: CC BY-NC-SA 3.0 IGO

5 Donadini MP, Ageno W. Should alcohol be considered a minor protective factor for unprovoked venous thromboembolism? Thromb Haemost 2013;110(01):2-3

6 Johansson M, Johansson L, Wennberg M, Lind M. Alcohol consumption and risk of first-time venous thromboembolism in men and women. Thromb Haemost 2019;119(06):962-970

7 Folsom AR, Lutsey PL, Roetker NS, et al. Elevated hepatic enzymes and incidence of venous thromboembolism: a prospective study. Ann Epidemiol 2014;24(11):817-821

8 Harrington LB, Hagan KA, Mukamal KJ, et al. Alcohol consumption and the risk of incident pulmonary embolism in US women and men. J Thromb Haemost 2018;16(09):1753-1762

9 Gregson J, Kaptoge S, Bolton T, et al; Emerging Risk Factors Collaboration. Cardiovascular risk factors associated with venous thromboembolism. JAMA Cardiol 2019;4(02):163-173

10 Gaborit FS, Overvad K, Nørgaard M, Kristensen SR, Tjønneland A, Severinsen MT. Alcohol intake and risk of venous thromboembolism. A Danish follow-up study. Thromb Haemost 2013;110(01): 39-45

11 Wattanakit K, Lutsey PL, Bell EJ, et al. Association between cardiovascular disease risk factors and occurrence of venous thromboembolism. A time-dependent analysis. Thromb Haemost 2012;108(03):508-515

12 Taylor-Robinson D, Kee F. Precision public health-the Emperor's new clothes. Int J Epidemiol 2018;48:1-6

13 Criqui MH, Thomas IC. Alcohol consumption and cardiac disease: where are we now? J Am Coll Cardiol 2017;69(01):25-27 